\title{
Establishment of the Seoul National University Prospectively Enrolled Registry for Genitourinary Cancer (SUPER-GUC): A prospective, multidisciplinary, bio-bank linked cohort and research platform
}

\author{
Chang Wook Jeong ${ }^{1}$, Jungyo Suh ${ }^{1}$, Hyeong Dong Yuk ${ }^{1,2}$, Bum Sik Tae ${ }^{1,3}$, Miso Kim ${ }^{4}$, Bhumsuk Keam ${ }^{4}$, Jin Ho Kim ${ }^{5}$, \\ Sang Youn $\mathrm{Kim}^{6}$, Jeong Yeon $\mathrm{Cho}^{6}$, Seung Hyup Kim ${ }^{6}$, Kyung Chul Moon ${ }^{7}$, Gi Jeong Cheon ${ }^{8}$, Ja Hyeon Ku${ }^{1}$, \\ Hyeon Hoe Kim ${ }^{1}$, Cheol Kwak \\ Departments of ${ }^{7}$ Urology, ${ }^{5}$ Radiation Oncology, ${ }^{6}$ Radiology, ${ }^{7}$ Pathology, and ${ }^{8}$ Nuclear Medicine, Seoul National University Hospital, Seoul National University College of \\ Medicine, Seoul, ${ }^{2}$ Department of Urology, Inje University Sanggye Paik Hospital, Seoul, ${ }^{3}$ Department of Urology, Korea University Ansan Hospital, Korea University College \\ of Medicine, Ansan, ${ }^{4}$ Department of Internal Medicine, Seoul National University Hospital, Cancer Research Institute, Seoul National University College of Medicine, Seoul, \\ Korea
}

Purpose: To establish a prospective, comprehensive, multidisciplinary, bio-bank linked genitourinary cancer cohort based on standard real practice.

Materials and Methods: We established the Seoul National University Prospectively Enrolled Registry for Genitourinary Cancer (SUPER-GUC), a prospective cohort clinical database and bio-specimen repository system for prostate cancer (SUPER-PC), renal cell carcinoma (SUPER-RCC), and urothelial cancer (SUPER-UC) at a high-volume, tertiary institution. Each cohort consists of several sub-cohorts based on treatment or disease status. Detailed longitudinal clinical information, and general and disease specific patient-reported outcomes are captured. We use the same evaluation format and questionnaires for all participating departments. Patients' blood, urine, tumor, and normal tissues are collected. The number of registered patients and their basic characteristics are summarized. For the surgical sub-cohort, study participation, bio-specimen, and tissue banking rates are analyzed.

Results: Since March 2016, 11 sub-cohorts for all disease statuses have been opened, ranging from low-risk localized to metastatic disease. SUPER-PC, SUPER-RCC, and SUPER-UC enrolled 929, 796, and 1,221 patients, respectively. Study participation, biosampling, and fresh frozen tumor banking rates of surgical sub-cohorts were $89.0 \%$ to $93.1 \%, 91.2 \%$ to $99.1 \%$, and $56.9 \%$ to $79.1 \%$, respectively.

Conclusions: SUPER-GUC is a study platform for comparative outcome, quality-of-life, and translational (genetics, biomarkers) research for genitourinary cancer.

Keywords: Biological specimen banks; Cohort studies; Prospective studies; Urogenital neoplasms

This is an Open Access article distributed under the terms of the Creative Commons Attribution Non-Commercial License (http://creativecommons.org/licenses/by-nc/4.0) which permits unrestricted non-commercial use, distribution, and reproduction in any medium, provided the original work is properly cited.

Received: 7 February, 2019 - Accepted: 19 March, 2019

Corresponding Author: Cheol Kwak

Department of Urology, Seoul National University Hospital, Seoul National University College of Medicine, 101 Daehak-ro, Jongno-gu, Seoul 03080, Korea

TEL: +82-2-2072-2999, FAX: +82-2-742-4665, E-mail: mdrafael@snu.ac.kr

ORCID: https://orcid.org/0000-0002-1987-2111 


\section{INTRODUCTION}

Genitourinary cancers are commonly diagnosed malignancies worldwide and significantly impact human health. Prostate cancer (PC) is the most commonly diagnosed malignancy in men in the United States and second most frequent cancer in men worldwide in 2018 [1,2]. Bladder and kidney cancers are globally the sixth and ninth most common cancers, respectively, in men [1] European countries have the highest incidence of these all three genitourinary cancers [1,3]. Furthermore, the incidence and mortality of these three genitourinary cancers have increased 2.5- and 1.6fold between 1990 and 2013, respectively [4]. This substantial increasing trend of global genitourinary cancer burden has not subsided.

While randomized controlled trials (RCTs) provide the highest level of evidence, they are not always feasible and sometimes impossible [5]. Narrow eligibility criteria and highly controlled settings of RCTs can rather limit their applicability [5]. Alternatively, high-quality cohort studies sometimes generate valuable information that is generally applicable, even in situations in which RCT is impractical [6]

Accordingly, we aimed to develop a prospective, comprehensive, multidisciplinary, bio-bank-linked cohort based on standard real practice for PC, renal cell carcinoma (RCC), and urothelial cancer (UC). After preparing for three years, we established the Seoul National University Prospectively Enrolled Registry for Genitourinary Cancer (SUPERGUC) in March 2016. The major goals of the SUPERGUC are as follows: 1) to conduct comparative outcome studies between various treatment options, 2) to identify prognostic factors for disease progression and mortality, 3) to facilitate various type of translational research including genetic or molecular profiling and biomarker studies, 4) to understand patients' quality of life (QoL) and promote QoL research including a patient-oriented outcome study and comparative-effectiveness research [7], 5) to investigate changes in body composition and its impacts on outcomes, and 6) to understand the influence of aging and fragility on treatment and cancer in elderly patients.

The present paper describes the design and methodology of SUPER-GUC. Furthermore, we summarize current patient enrollment status and basic demographics.

\section{MATERIALS AND METHODS}

\section{Ethics statement}

All study protocols for SUPER-PC, SUPER-RCC, and SUPER-UC were approved by the Institutional Review
Board (IRB) of Seoul National University Hospital (Seoul, Korea). The approval numbers are H-1506-121-682, H-1506-120682, and H-1506-122-682, respectively. Informed consent was obtained by all subjects when they were enrolled.

\section{Organization}

As faculty providing direct treatment, four uro-oncologists, two medical oncologists, and one radiation oncologist, who mainly manage treat genitourinary cancer patients, participate with this cohort. They exclusively manage almost all genitourinary cancer patients in our tertiary high-volume institution. As supporting faculty, three uroradiologists, one nuclear medicine physician, and one uropathologist exclusively interpret medical images and pathological results. Participating faculty are all wellexperienced, dedicated specialists in their field. We have one permanent information technology (IT) engineer consultant and three IT advisors.

\section{Sub-cohorts and eligibility criteria}

SUPER-GUC consists of three cohorts based on cancer type, SUPER-PC, SUPER-RCC, and SUPER-UC. Each cohort is composed of several sub-cohorts based on treatment or disease status. Names of the sub-cohorts and their eligibility criteria are described in Table 1.

\section{Patient process, data collection, and bio-specimen repository}

In principle, the cohort is based on real clinical practice, and the study does not interfere with actual practice. All patients are enrolled following provision of written informed consent for the cohort study based on cancer type as well as for the additional bio-specimen registry.

All data were collected using the Research Electronic Data Capture (REDCap) system in an independent server with a triple back-up system. REDCap is a secure, web-based application designed to support data capture for research studies [8]. Electronic case-report forms (eCRF) consist of several forms (designated as 'instruments' in REDCap), and selected forms can be repeatedly entered to capture longitudinal data. Furthermore, some common forms are shared by all sub-cohorts in SUPER-GUC. We categorized data entering forms as once entering, repeatedly entering, and revisingly entering forms. After entering all data in once entering type form, it is finalized and locked. We can handle repeating data using repeatedly entering forms, such as questionnaires or transurethral resection of bladder tumor information. In revisingly entering forms, we can revise data until it is finalized. For instance, the last follow- 
Table 1. Sub-cohorts of the SUPER-GUC and their eligibility criteria

\begin{tabular}{|c|c|}
\hline Cohort & Eligibility criteria \\
\hline \multicolumn{2}{|l|}{ SUPER-PC } \\
\hline SUPER-PC-RP & Pathologically proven prostate cancer patients who undergo radical prostatectomy \\
\hline SUPER-PC-AS & $\begin{array}{l}\text { Pathologically proven low risk prostate cancer patients within } 6 \text { months who undergo active surveillance as initial } \\
\text { treatment. They should be eligible by strict criteria registered in ClinicalTrials.gov protocol (NCT02971085), when patients } \\
\text { want and meet usual active surveillance criteria they can be enrolled as non-strict criteria. }\end{array}$ \\
\hline SUPER-PC-RT & $\begin{array}{l}\text { Pathologically proven prostate cancer patients who undergo radiation therapy with curative intent. It includes primary, } \\
\text { adjuvant and salvage radiation therapy, but palliative radiation therapy is not eligible. }\end{array}$ \\
\hline SUPER-PC-HSPC & $\begin{array}{l}\text { Pathologically proven non-metastatic or metastatic hormone sensitive prostate cancer patients who undergo medical } \\
\text { therapy as major initial treatment. It includes watchful waiting, ADT and chemo-ADT. Neoadjuvant or adjuvant medical } \\
\text { therapy for other major treatment is not eligible. }\end{array}$ \\
\hline SUPER-PC-CRPC & Pathologically proven prostate cancer patients, who were progressed as CRPC within 3 months. \\
\hline \multicolumn{2}{|l|}{ SUPER-RCC } \\
\hline SUPER-RCC-Nx & $\begin{array}{l}\text { Clinically diagnosed as renal cell carcinoma patients who undergo surgical treatment. It includes ablation, partial or radical } \\
\text { nephrectomy. It is not limited to localized cancer, thus cytoreductive nephrectomy in metastatic disease is also eligible. }\end{array}$ \\
\hline SUPER-RCC-mRCC & $\begin{array}{l}\text { Pathologically proven renal cell carcinoma patients who were diagnosed as advanced (T4 or N1) or metastatic disease } \\
\text { within } 3 \text { months and undergo initial treatment for advanced or metastatic RCC. Nephrectomy or metastasectomy before } \\
\text { pathologic diagnosis does not limit the enrollment. }\end{array}$ \\
\hline \multicolumn{2}{|l|}{ SUPER-UC } \\
\hline SUPER-UC-TURB & Clinically diagnosed as bladder cancer patients who undergo transurethral resection of bladder tumor. \\
\hline SUPER-UC-CX & Pathologically proven bladder cancer patients who undergo radical cystectomy. \\
\hline SUPER-UC-UTUC & $\begin{array}{l}\text { Clinically diagnosed as upper urinary tract urothelial cancer patients who undergo curative surgical treatment for it. It } \\
\text { includes nephroureterectomy, segmental ureterectomy and any kind of nephron sparing endoscopic treatment. It also } \\
\text { includes definitive treatment plan after intraoperative decision by ureteroscopy with or without frozen biopsy. }\end{array}$ \\
\hline SUPER-UC-mUC & $\begin{array}{l}\text { Pathologically proven urothelial cancer patients who were diagnosed as advanced (non-localized or lymph node involve- } \\
\text { ment) or metastatic disease within } 3 \text { months and undergo initial treatment for advanced or metastatic urothelial cancer. }\end{array}$ \\
\hline
\end{tabular}

SUPER, Seoul National University Prospectively Enrolled Registry; GUC, Genitourinary Cancer; PC, prostate cancer; RP, radical prostatectomy; AS, active surveillance; RT, radiation therapy; HSPC, hormone-sensitive prostate cancer; ADT, androgen deprivation therapy; CRPC, castrationresistant prostate cancer; $\mathrm{RCC}$, renal cell carcinoma; Nx, nephrectomy; mRCC, metastatic renal cell carcinoma; UC, urothelial cancer; TURB, transurethral resection of bladder tumor; Cx, cystectomy; UTUC, upper urinary tract urothelial cancer; mUC, metastatic urothelial cancer.

up date is revised following every hospital visit in this of form.

We constructed eCRF forms to be as detailed as possible. For example, SUPER-PC-Radical Prostatectomy (RP) has 20 forms with 784 fields. Table 2 shows construction of the eCRF for SUPER-PC-RP. Fig. 1 depicts the exemplary data collection process for SUPER-PC-RP with eCRFs. Each subcohort has its own predefined data collection process.

We selected questionnaires according to two principles. First, the Korean version should be validated and easily incorporated in daily practice. Some questionnaires were already validated, and some were validated by our team for SUPER-GUC. For measuring health-related utility and general QoL, we universally use EQ-5D-5L across all subcohorts. In addition, we use the International Prostate Symptom Score (IPSS), International Index of Erectile Function-5 (IIEF-5), and Expanded Prostate Cancer Index Composite for Clinical Practice (EPIC-CP) for PC patients. The 15-item Functional Assessment of Cancer TherapyKidney Symptom Index (FKSI-15) and the Functional
Assessment of Cancer Therapy-Vanderbilt Cystectomy Index (FACT-VCI) were selected as disease-specific questionnaires for RCC and cystectomy patients, respectively. We also use the Convalescence And Recovery Evaluation (CARE) questionnaire to evaluate short-term ( $\leq 3$ months) QoL after major surgery [9]. The G8 geriatric screening tool is used for patients who are 65 years or older [10,11].

Surgical complications are evaluated and collected using the revised Clavien-Dindo system and European Association of Urology (EAU) guidelines [12,13]. All treatment-related adverse events are evaluated with the same forms of the Common Terminology Criteria for Adverse Events (CTCAE) ver. 4.0.3. Regardless of treating departments, we standardized all evaluation formats, including questionnaires and adverse events assessment.

To measure body composition based on bioelectrical impedance analysis (BIA), we use the InBody 570 (InBody $\mathrm{Co}$, Ltd, Seoul, Korea). This is a well-established method to determine body composition such as lean muscle mass and body fat mass [14-16]. 
Table 2. Architecture of electronic case-report forms for Seoul National University Prospectively Enrolled Registry for Prostate Cancer-Radical Prostatectomy (SUPER-PC-RP)

\begin{tabular}{|c|c|c|c|}
\hline Name of form & Number of fields & Entering type & Description and examples \\
\hline Study data \& demographics & 39 & Once entering & Study number, basic demographics and bio-banking information \\
\hline Preop. characteristics & 54 & Once entering & Initial PSA, biopsy information, past medical, social and family history \\
\hline Preop. imaging & 19 & Once entering & Results of magnetic resonance imaging and bone scan \\
\hline Operation & 38 & Once entering & Details of surgery \\
\hline Pathology & 50 & Once entering & Final pathology details \\
\hline Postop. outcomes & 9 & Once entering & $\begin{array}{l}\text { Short-term postoperative results such as duration of hospital stay, } \\
\text { cystography and Foley catheter removal date }\end{array}$ \\
\hline Complication & 31 & Once entering & Intraoperative, immediate, 30 and 90 days complications \\
\hline Lab & 35 & Repeatedly entering & Laboratory results (except PSA) \\
\hline PSA & 202 & Revisingly entering & PSA results \\
\hline Medication \& comorbidity & 22 & Repeatedly entering & Information of medication and comorbidity \\
\hline$A / E$ & 62 & Repeatedly entering & Post-treatment adverse events \\
\hline IPSS & 14 & Repeatedly entering & International Prostate Symptom Score questionnaire \\
\hline EPIC-CP & 26 & Repeatedly entering & $\begin{array}{l}\text { Expanded Prostate Cancer Index Composite for Clinical Practice } \\
\text { questionnaire }\end{array}$ \\
\hline IIEF-5 & 9 & Repeatedly entering & International Index of Erectile Function-5 questionnaire \\
\hline EQ-5D-5L & 10 & Repeatedly entering & EuroQol health-related utility measurement tool \\
\hline CARE questionnaire & 35 & Repeatedly entering & Convalescence And Recovery Evaluation questionnaire \\
\hline G8 & 10 & Once entering & Geriatric assessment by the $\mathrm{G} 8$ \\
\hline InBody & 26 & Repeatedly entering & Body composition measurement by bio-electrical impedance analysis \\
\hline Functional outcome summary & 30 & Revisingly entering & Summarized results of continence and potency \\
\hline Oncologic outcome summary & 63 & Revisingly entering & Adjuvant or salvage treatment and oncologic outcome results \\
\hline
\end{tabular}

Preop., preoperative; postop., postoperative; PSA, prostate-specific antigen.

We collect patients' pre-treatment whole blood, serum, buffy coat, plasma, and urine samples at the time of entering new sub-cohorts. Collected samples are divided in microvials and stored in a $-80^{\circ} \mathrm{C}$ deep freezer or $-195^{\circ} \mathrm{C}$ liquid nitrogen container according to the sample type at the Seoul National University Hospital Human Biobank. Tumor and normal tissues are collected in the operating room or frozen biopsy room up to six vials each and immediately stored in a $-195^{\circ} \mathrm{C}$ liquid nitrogen tank at the Seoul National University Hospital Cancer Tissue Bank. These two banking systems are qualified, centralized bio-banking systems at our institution that have been in operation for almost a decade. Formalin-fixed, paraffin-embedded tissue blocks are available in virtually $100 \%$ patients managed by the Department of Pathology.

\section{Outcome measurements}

A major point of interest regarding the cohort is oncologic outcomes. Baseline cancer status and general condition are evaluated at the time of enrollment for each sub-cohort. Treatment response, time of recurrence, and survival are evaluated. Furthermore, baseline cancer status and oncologic outcome are repeatedly evaluated for every single major treatment in advanced or metastatic disease. Date and cause of death are confirmed by regular linkage with death registry at the Statistics Korea.

Functional outcomes measured in PC patients include urinary and sexual function. For the nephrectomy subcohort, renal function was also determined using estimated glomerular filtration rate or renal scan and progression to end-stage renal disease.

\section{Statistical analysis}

We summarize current enrollment status, basic demographics, and sampling information. Data are expressed as the mean \pm standard deviation. A p-values $<0.05$ were considered statistically significant. All statistical analyses were performed using $\mathrm{R}$ for Windows, version 3.5.1 (http:// www.r-project.org).

\section{RESULTS}

The first patient was enrolled March 7, 2016 in SUPERPC. At the end of December, 2018, a total of 929, 796, and 1,221 patients were enrolled in SUPER-PC, SUPER-RCC, and SUPER-UC, respectively. Results of patient enrollment 


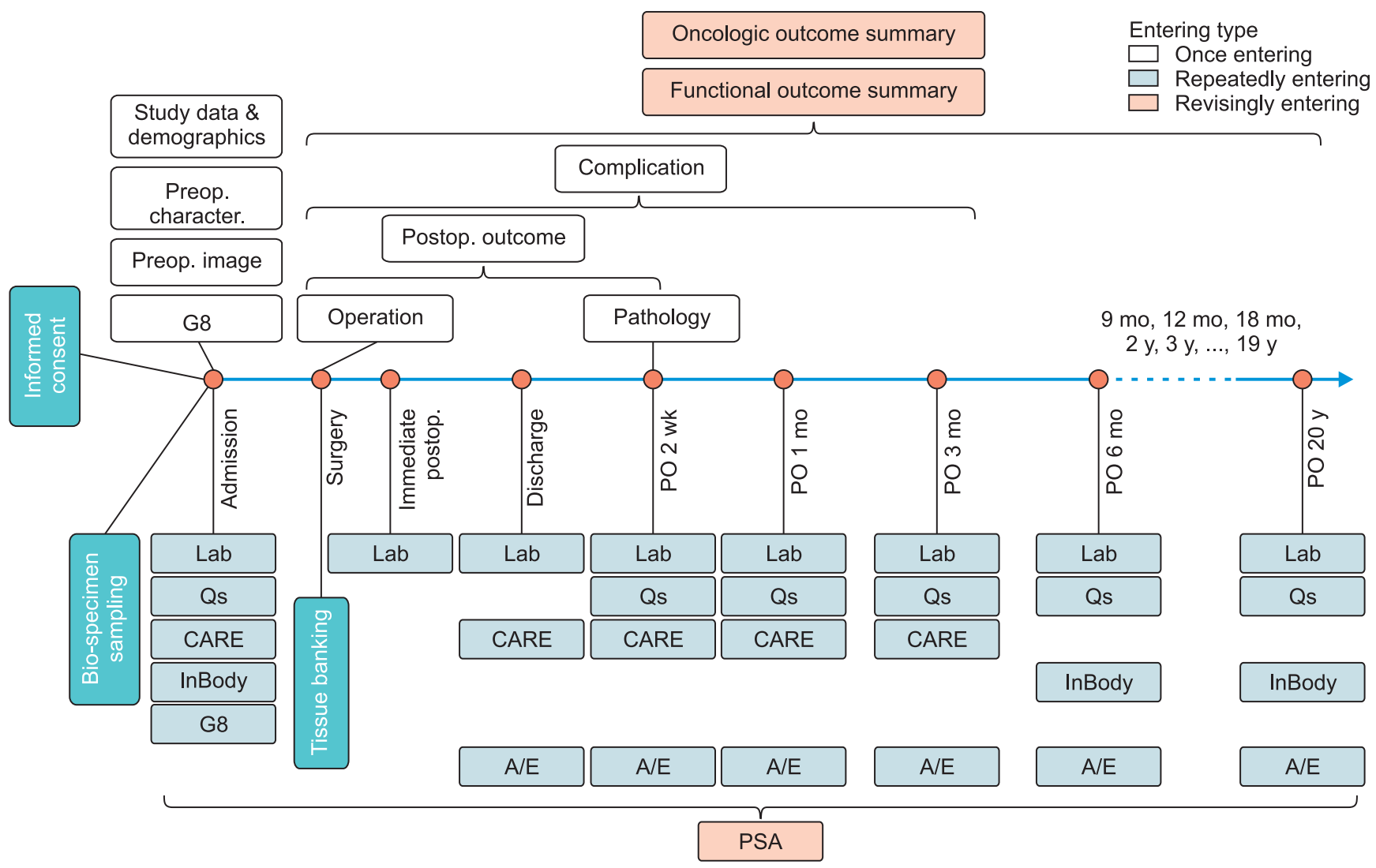

Fig. 1. Data collection process for the Seoul National University Prospectively Enrolled Registry for Prostate Cancer-Radical Prostatectomy (SUPERPC-RP) with electronic case report forms. Preop., preoperative; character., characteristics; postop., postoperative; PO, postoperative; Qs, questionnaires; CARE, Convalescence And Recovery Evaluation; A/E, adverse events; PSA, prostate-specific antigen.

Table 3. Patient enrollment status of Seoul National University Prospectively Enrolled Registry for Genitourinary Cancer (SUPER-GUC) at the end of December, 2018

\begin{tabular}{llc}
\hline \multicolumn{1}{c}{ Cohort } & First enrollment date & Number of patients \\
\hline SUPER-PC & & 929 \\
SUPER-PC-RP & March 6, 2016 & 765 \\
SUPER-PC-AS & December 23, 2016 & 56 \\
SUPER-PC-RT & May 20, 2016 & 39 \\
SUPER-PC-HSPC & October 18, 2016 & 56 \\
SUPER-PC-CRPC & April 3, 2017 & 13 \\
SUPER-RCC & & 796 \\
SUPER-RCC-Nx & March 10, 2016 & 762 \\
SUPER-RCC-mRCC & June 2, 2016 & 34 \\
SUPER-UC & & 1,221 \\
SUPER-UC-TURB & March 21, 2016 & 859 \\
SUPER-UC-CX & March 21, 2016 & 170 \\
SUPER-UC-UTUC & March 7, 2016 & 163 \\
SUPER-UC-mUC & March 28, 2016 & 29 \\
\hline
\end{tabular}

SUPER, Seoul National University Prospectively Enrolled Registry; PC, prostate cancer; RP, radical prostatectomy; AS, active surveillance; RT, radiation therapy; HSPC, hormone-sensitive prostate cancer; CRPC, castration-resistant prostate cancer; RCC, renal cell carcinoma; Nx, nephrectomy; mRCC, metastatic renal cell carcinoma; UC, urothelial cancer; TURB, transurethral resection of bladder tumor; Cx, cystectomy; UTUC, upper urinary tract urothelial cancer; mUC, metastatic urothelial cancer. are summarized in Table 3. Table 4 shows rates of study participation, bio-sampling, and fresh frozen tumor banking and baseline characteristics of surgical sub-cohorts.

For SUPER-PC-RP, we also developed an online interactive data visualization and analysis platform using Tableau Server ver. 10.3.1 (Tableau Software, Inc, Seattle, WA, USA) (Fig. 2) $[17,18]$. Results are interactively presented in real time as we change the dimensional variables.

\section{DISCUSSION}

There are several types of prospective cohort or registries [19]. Population-based cohorts systematically collect data on all or randomly selected patients with a certain disease in a given geographic area within a given time [20]. They usually link with national claims data and statistics [21]. Thus, representativeness and generalizability are the advantage, while incomplete or inaccurate data coding and lack of detailed information are potential serious pitfalls [21]. Community and/or multicenter cohorts may be the most common form of cohorts [19]. A greater number of involved institutions increases the generalizability. However, relatively high cost, standardization between 
Table 4. Participation, banking rates, and baseline characteristics of surgical sub-cohorts in SUPER-GUC

\begin{tabular}{|c|c|c|c|c|c|}
\hline Characteristic & SUPER-PC-RP & SUPER-RCC-Nx & SUPER-UC-TURB & SUPER-UC-CX & SUPER-UC-UTUC \\
\hline No. of patients enrolled & 765 & 762 & 859 & 170 & 163 \\
\hline Participation rate ${ }^{a}$ & $765 / 836(91.5)$ & $762 / 853(89.3)$ & $859 / 964(89.1)$ & $170 / 191(89.0)$ & $163 / 175(93.1)$ \\
\hline Bio-specimen sampling rate & 758/765 (99.1) & $733 / 762(96.2)$ & $829 / 859(96.5)$ & $155 / 170(91.2)$ & $155 / 163(95.1)$ \\
\hline Tumor banking & $463 / 765(60.5)$ & $565 / 762(74.1)$ & $489 / 859(56.9)$ & $117 / 170(68.8)$ & $129 / 163(79.1)$ \\
\hline \multicolumn{6}{|l|}{ Baseline characteristics } \\
\hline Age (y) & $68.7 \pm 6.6$ & $59.4 \pm 12.4$ & $68.9 \pm 11.9$ & $67.0 \pm 10.9$ & $68.5 \pm 9.9$ \\
\hline \multicolumn{6}{|l|}{ Sex } \\
\hline Male & $765(100.0)$ & $521(68.4)$ & $687(80.0)$ & $128(75.3)$ & $113(69.3)$ \\
\hline Female & $0(0.0)$ & $241(31.6)$ & $172(20.0)$ & $42(24.7)$ & $50(30.7)$ \\
\hline Clinical T stage & & & $\mathrm{b}$ & & \\
\hline$\leq \mathrm{T} 1$ & $674(88.1)$ & $658(86.4)$ & $568(66.1)$ & $59(34.7)$ & $123(75.5)$ \\
\hline $\mathrm{T} 2$ & $79(10.3)$ & $63(8.3)$ & $86(10.0)^{c}$ & $88(51.8)$ & $17(10.4)$ \\
\hline T3 & $12(1.6)$ & $38(5.0)$ & - & $15(8.8)$ & $22(13.5)$ \\
\hline T4 & $0(0.0)$ & $3(0.4)$ & - & $8(4.7)$ & $1(0.6)$ \\
\hline Tx & $0(0.0)$ & $0(0.0)$ & $205(23.9)^{d}$ & $0(0.0)$ & $0(0.0)$ \\
\hline \multicolumn{6}{|l|}{ Clinical N stage } \\
\hline No & 672 (87.8) & 738 (96.9) & - & $145(85.3)$ & $145(89.0)$ \\
\hline $\mathrm{N}+$ & $86(11.2)$ & $24(3.1)$ & - & $25(14.7)$ & $18(11.0)$ \\
\hline $\mathrm{Nx}$ & $7(0.9)$ & $0(0.0)$ & - & $0(0.0)$ & $0(0.0)$ \\
\hline \multicolumn{6}{|l|}{ Clinical M stage } \\
\hline M0 & 738 (96.5) & 738 (96.9) & - & 169 (99.4) & $162(99.4)$ \\
\hline $\mathrm{M}+$ & $0(0.0)$ & $24(3.1)$ & - & $1(0.6)$ & $1(0.6)$ \\
\hline$M x$ & $27(3.5)$ & $0(0.0)$ & - & $0(0.0)$ & $0(0.0)$ \\
\hline
\end{tabular}

Values are presented as number only, number/total number (\%), mean \pm standard deviation, or number (\%).

SUPER, Seoul National University Prospectively Enrolled Registry; GUC, Genitourinary Cancer; PC, prostate cancer; RP, radical prostatectomy; RCC, renal cell carcinoma; Nx, nephrectomy; UC, urothelial cancer; TURB, transurethral resection of bladder tumor; Cx, cystectomy; UTUC, upper urinary tract urothelial cancer.

${ }^{\mathrm{a}}$ :Percentage of patients enrolled among all eligible patients, ${ }^{\mathrm{b}}:$ pathologic stage of first TURB after enrolled, ${ }^{\mathrm{c}}:$ pathologic stage $\geq \mathrm{T} 2{ }^{\mathrm{d}}:$ :pathologic T0 or benign.

institutions, and maintaining high quality are ongoing challenges. Single institutional cohort may be limited by its lack of representativeness; however, it has the advantage of being able to collect additional detailed information [19]. Furthermore, it is relatively easy to maintain high quality.

Traditionally, numerous studies were conducted as retrospective single center case series, rather than an actual cohort $[6,19,22]$. Occasionally, the reports insisted they used a prospectively collected database, but they were usually limited by many biases and missing data. Thus, we initiated SUPER-GUC as a prospective, single institutional cohort with flexible structure; this can be extended as multicenter cohort in the future. Additionally, although we started it as a single institutional cohort, we endeavored to overcome limitation of single-center cohort and maximize its quality and potential for utilization.

First, we organized it as multidisciplinary cohort, which collects data from all related departments using standardized methods. We also unified data collection forms and timing, especially QoL questionnaires and adverse events assessment. Patients can select different treatment provided by different specialties, even for the same disease status. For example, localized PC patients may undergo either RP provided by a urologist or radiation therapy provided by a radiation oncologist [23]. Furthermore, patients may have sequential treatments through different departments dependent upon disease progression. For instance, after radical cystectomy performed by a urologist, adjuvant or palliative chemotherapy can be provided by a medical oncologist. Based on this multidisciplinary endeavor, we can compare any treatment outcome as well as that of any follow-up evaluation throughout long-term treatment without cessation.

Next, we constructed a comprehensive cohort consisting of sub-cohorts for all possible disease conditions and treatments. Patients may be entered to a specific sub-cohort from other sub-cohorts dependent upon disease progression; alternatively, newly referred patients may be enrolled 
A

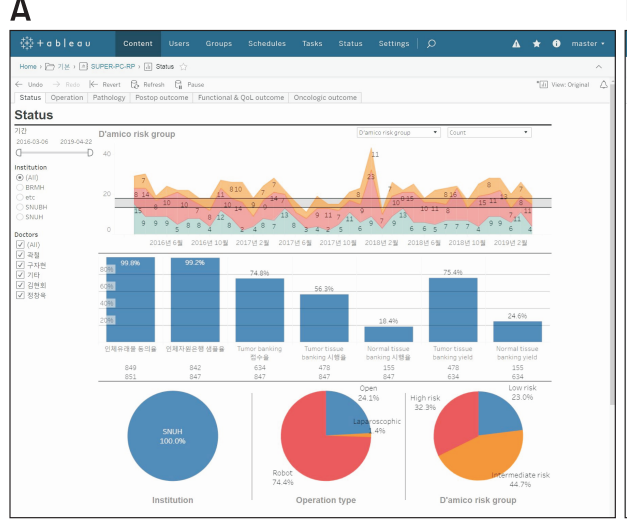

C

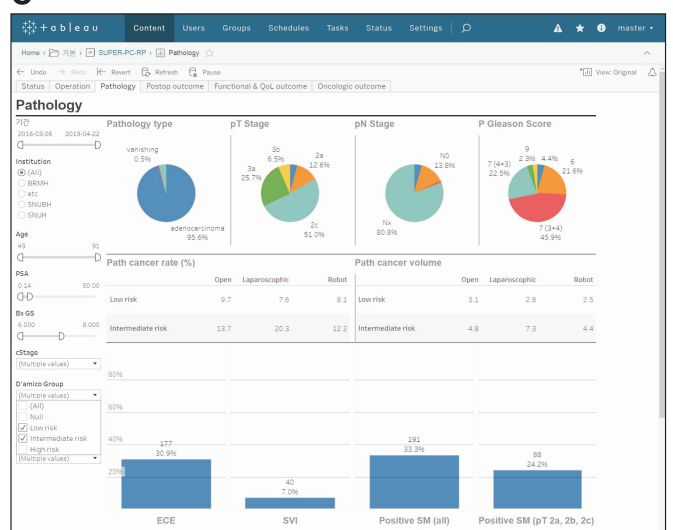

B

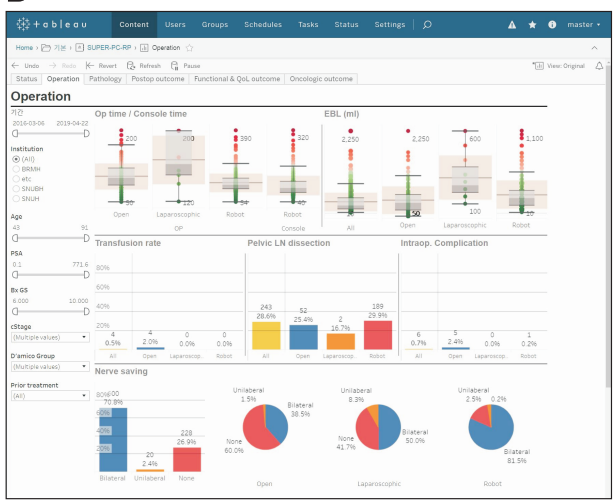

D

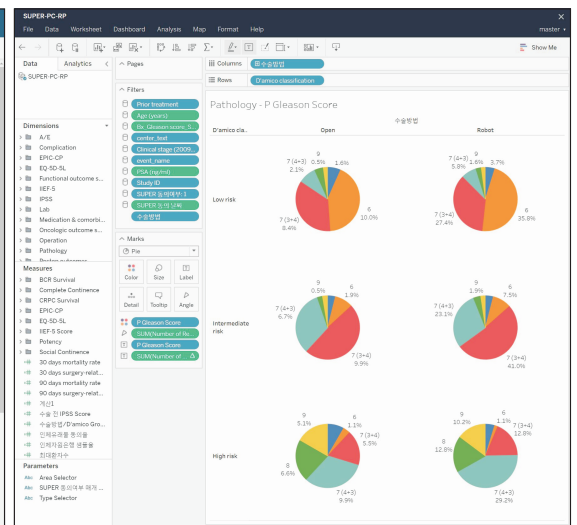

Fig. 2. Image captures of online interactive data visualization and analysis platform for Seoul National University Prospectively Enrolled Registry for Prostate Cancer-Radical Prostatectomy (SUPERP(-RP). Dashboards are interactively changed when dimensional variables are changed in left panel. (A) Enrollment status, (B) operative methods and results, (C) pathologic outcomes, (D) example of interactive analysis. Pathologic Gleason Score by operative type and D'Amico classification. directly to the sub-cohort. This sub-cohort structure allows us to effectively recruit patients, especially those with a more advanced condition, and collect specific detailed data focusing on the status of each patient. The Cancer of the Prostate Strategic Urologic Research Endeavor (CaPSURE) is one of the most successful prospective PC cohorts [19,24]. This cohort recruited over 15,000 patients at all stages from 43 urology practices for three decades [19,24,25]. However, only newly diagnosed PC patients are eligible in a singlephase design [24,25]. Therefore, localized cancer and initial treatment are highly focused, whereas data after progression or later phase treatment (e.g., androgen receptor-targeted treatment for castration-resistant PC) are limited. We expect we can overcome this using our comprehensive, multi-subcohort design.

Third, the bio-specimen repository is another important feature of SUPER-GUC. Traditionally, all formalin-fixed, paraffin-embedded tissue blocks of surgical specimens are stored in our institution. However, we extend this by collecting pre-treatment blood and urine in most cases and freshly frozen tumor and normal tissues in all possible cases. These bio-specimens are stored and managed by qualified, centralized bio-banking systems that are certified by the government. Thus, SUPER-GUC can support a variety of translational research, such as biomarker or genetic profiling studies. For instance, we are currently conducting a study to evaluate the association between congenital immune deficiency with cancer characteristics and outcomes in 540 bladder cancer patients. Several urine biomarker studies for bladder cancer were conducted or are currently in progress. We also performed comprehensive genetic characterization of transcription factor E3 (TFE3)-overexpressed RCC using the SUPER-RCC cohort. Recently, we conducted a predictive serum biomarker study that differentiates benign tumors from RCC using SUPER-RCC-Nephrectomy (Nx). We are cautiously confident that SUPER-GUC will be an important translational research platform.

Fourth, even though it is institutional cohort, mortality information is regularly collected from the government death registry at the Statistics Korea. Accordingly, we can assure survival data, the most important outcome of SUPER-GUC.

Finally, we collect a variety of data for comprehensive future studies. Using validated instruments, patient-reported health-related QoL are evaluated. Since usual health-related QoL questionnaires cannot sensitively capture differences in QoL between surgery types, we also collect CARE questionnaire before and within 3 months after operation [9]. 
To evaluate the effect of vulnerability, we also use the G8 geriatric screening tool for elderly patients for all treatments [10,11]. Moreover, to more precisely measure muscle and fat mass and determine their association with outcome, we regularly measure body composition using BIA [14-16].

SUPER-GUC also has several limitations. Since it is a single-center cohort, it is difficult to enroll a large patient number and difficult to represent whole population. However, its flexible design and open structure can expend SUPER-GUC for multi-center collaboration. Actually, SUPER-PC-Active Surveillance (AS) is being adapted for a nationwide, multicenter cohort of the Korean Urological Oncology Society by reducing variables and restructuring the eCRF. Because non-surgical sub-cohorts were opened later, numbers of patients in these sub-cohorts are relatively small. An outpatient-based patient recruiting system has been recently established. Therefore, we expect a rapid increase in the size of non-surgical sub-cohorts. Furthermore, although inherent bias from an observational study is inevitable, it may be minimized using a recent statistical adjusting methodology $[6,26,27]$.

There are many prospective cohorts in the uro-oncology field; however, they are for specific cancer types and are generally limited to specific disease conditions or treatments [19,24,28-30]. To our knowledge, SUPER-GUC is the first comprehensive, prospective, bio-bank-linked cohort covering all major genitourinary cancers for all disease statuses and treatments.

\section{CONCLUSIONS}

In summary, we successfully established a comprehensive, prospective, multidisciplinary, bio-bank-linked genitourinary cancer cohort, SUPER-GUC. It will support a variety of research regarding comparative outcomes, QoL studies, and translational research such as genetic or biomarker studies for prostate, kidney, and UCs.

\section{CONFLICTS OF INTEREST}

The authors have nothing to disclose.

\section{ACKNOWLEDGMENTS}

This study was supported by grants from the National R\&D Program for Cancer Control (HA17C0039) and the Korea Health Technology R\&D Project (HI14C2404) through the Korea Health Industry Development Institute (KHIDI), funded by the Ministry of Health \& Welfare, Republic of
Korea. None of the sponsors had any access to the data or any influence on or access to the analysis plan, the results, or the manuscript.

The bio-specimens for this study were managed by the Seoul National University Hospital Cancer Tissue Bank and the Seoul National University Hospital Human Biobank, a member of the Korea Biobank Network, which is supported by the Ministry of Health and Welfare. All samples derived from these banking systems were obtained with informed consent under institutional review board-approved protocols.

\section{REFERENCES}

1. Bray F, Ferlay J, Soerjomataram I, Siegel RL, Torre LA, Jemal A. Global cancer statistics 2018: GLOBOCAN estimates of incidence and mortality worldwide for 36 cancers in 185 countries. CA Cancer J Clin 2018;68:394-424.

2. Siegel RL, Miller KD, Jemal A. Cancer statistics, 2018. CA Cancer J Clin 2018;68:7-30.

3. Znaor A, Lortet-Tieulent J, Laversanne M, Jemal A, Bray F. International variations and trends in renal cell carcinoma incidence and mortality. Eur Urol 2015;67:519-30.

4. Dy GW, Gore JL, Forouzanfar MH, Naghavi M, Fitzmaurice C. Global burden of urologic cancers, 1990-2013. Eur Urol 2017;71:437-46.

5. Lavallée LT, Fergusson D, Breau RH. The role of randomized controlled trials in evidence-based urology. World J Urol 2011;29:257-63.

6. Yang W, Zilov A, Soewondo P, Bech OM, Sekkal F, Home PD. Observational studies: going beyond the boundaries of randomized controlled trials. Diabetes Res Clin Pract 2010;88 Suppl 1:S3-9.

7. Ahn S, Lee M, Jeong CW. Comparative quality-adjusted survival analysis between radiation therapy alone and radiation with androgen deprivation therapy in patients with locally advanced prostate cancer: a secondary analysis of Radiation Therapy Oncology Group 85-31 with novel decision analysis methods. Prostate Int 2018;6:140-4.

8. Harris PA, Taylor R, Thielke R, Payne J, Gonzalez N, Conde JG. Research electronic data capture (REDCap)--a metadatadriven methodology and workflow process for providing translational research informatics support. J Biomed Inform 2009;42:377-81.

9. Hollenbeck BK, Dunn RL, Wolf JS Jr, Sanda MG, Wood DP, Gilbert SM, et al. Development and validation of the convalescence and recovery evaluation (CARE) for measuring quality of life after surgery. Qual Life Res 2008;17:915-26.

10. Bellera CA, Rainfray M, Mathoulin-Pélissier S, Mertens C, Delva F, Fonck M, et al. Screening older cancer patients: first 
evaluation of the G-8 geriatric screening tool. Ann Oncol 2012;23:2166-72.

11. Decoster L, Van Puyvelde K, Mohile S, Wedding U, Basso U, Colloca G, et al. Screening tools for multidimensional health problems warranting a geriatric assessment in older cancer patients: an update on SIOG recommendations $†$. Ann Oncol 2015;26:288-300.

12. Dindo D, Demartines N, Clavien PA. Classification of surgical complications: a new proposal with evaluation in a cohort of 6336 patients and results of a survey. Ann Surg 2004;240:20513.

13. Mitropoulos D, Artibani W, Graefen M, Remzi M, Rouprêt M, Truss M; European Association of Urology Guidelines Panel. Reporting and grading of complications after urologic surgical procedures: an ad hoc EAU guidelines panel assessment and recommendations. Eur Urol 2012;61:341-9.

14. Gibson AL, Holmes JC, Desautels RL, Edmonds LB, Nuudi L. Ability of new octapolar bioimpedance spectroscopy analyzers to predict 4-component-model percentage body fat in Hispanic, Black, and White adults. Am J Clin Nutr 2008;87:332-8.

15. Maeda K, Koga T, Nasu T, Takaki M, Akagi J. Predictive accuracy of calf circumference measurements to detect decreased skeletal muscle mass and European Society for Clinical Nutrition and Metabolism-defined malnutrition in hospitalized older patients. Ann Nutr Metab 2017;71:10-5.

16. Koo BK, Kim D, Joo SK, Kim JH, Chang MS, Kim BG, et al. Sarcopenia is an independent risk factor for non-alcoholic steatohepatitis and significant fibrosis. J Hepatol 2017;66:123-31.

17. Ko I, Chang H. Interactive data visualization based on conventional statistical findings for antihypertensive prescriptions using National Health Insurance claims data. Int J Med Inform 2018;116:1-8.

18. Ko I, Chang H. Interactive visualization of healthcare data using tableau. Healthc Inform Res 2017;23:349-54.

19. Gandaglia G, Bray F, Cooperberg MR, Karnes RJ, Leveridge MJ, Moretti K, et al. Prostate cancer registries: current status and future directions. Eur Urol 2016;69:998-1012.

20. Parkin DM. The evolution of the population-based cancer registry. Nat Rev Cancer 2006;6:603-12.

21. Kang M, Ku JH, Kwak C, Kim HH, Jeong CW. Effects of aspirin, nonsteroidal anti-inflammatory drugs, statin, and COX2 inhibitor on the developments of urological malignancies: a population-based study with 10-year follow-up data in Korea. Cancer Res Treat 2018;50:984-91.

22. Kim BS, Tae BS, Ku JH, Kwak C, Kim HH, Jeong CW. Rate and association of lower urinary tract infection with recurrence after transurethral resection of bladder tumor. Investig Clin Urol 2018;59:10-7.

23. Mottet N, Bellmunt J, Bolla M, Briers E, Cumberbatch MG, De Santis M, et al. EAU-ESTRO-SIOG guidelines on prostate cancer. Part 1: screening, diagnosis, and local treatment with curative intent. Eur Urol 2017;71:618-29.

24. Cooperberg MR, Park S, Carroll PR. Prostate cancer 2004: insights from national disease registries. Oncology (Williston Park) 2004;18:1239-47; discussion 1248-50, 1256-8.

25. Lubeck DP, Litwin MS, Henning JM, Stier DM, Mazonson P, Fisk R, et al. The CaPSURE database: a methodology for clinical practice and research in prostate cancer. CaPSURE Research Panel. Cancer of the Prostate Strategic Urologic Research Endeavor. Urology 1996;48:773-7.

26. Nohr EA, Liew Z. How to investigate and adjust for selection bias in cohort studies. Acta Obstet Gynecol Scand 2018;97:40716.

27. Törner A, Dickman P, Duberg AS, Kristinsson S, Landgren O, Björkholm M, et al. A method to visualize and adjust for selection bias in prevalent cohort studies. Am J Epidemiol 2011;174:969-76.

28. Barocas DA, Alvarez J, Resnick MJ, Koyama T, Hoffman KE, Tyson MD, et al. Association between radiation therapy, surgery, or observation for localized prostate cancer and patientreported outcomes after 3 years. JAMA 2017;317:1126-40.

29. Thorsteinsdottir T, Stranne J, Carlsson S, Anderberg B, Björholt I, Damber JE, et al. LAPPRO: a prospective multicentre comparative study of robot-assisted laparoscopic and retropubic radical prostatectomy for prostate cancer. Scand J Urol Nephrol 2011;45:102-12.

30. Wijburg CJ, Michels CTJ, Oddens JR, Grutters JPC, Witjes JA, Rovers MM. Robot assisted radical cystectomy versus open radical cystectomy in bladder cancer (RACE): study protocol of a non-randomized comparative effectiveness study. BMC Cancer 2018;18:861. 